\title{
Single-arm open-label study of Durolane (NASHA nonanimal hyaluronic acid) for the treatment of osteoarthritis of the thumb
}

This article was published in the following Dove Press journal:

Open Access Rheumatology: Research and Reviews

27 March 2017

Number of times this article has been viewed

\section{Eloisa Velasco' $M^{a}$ Victoria Ribera ${ }^{2}$ \\ Joan $\mathrm{Pi}^{3}$}

'Department of Orthopedic Surgery, Hospital de Sant Joan Despí Moisés Broggi, Barcelona, Spain; ${ }^{2}$ Department of Anesthesiology, Vall d'Hebron University Hospital, Barcelona, Spain; ${ }^{3}$ Department of Orthopedics and Traumatology, Parc Taulí University Hospital, Sabadell, Barcelona, Spain

Correspondence: Eloisa Velasco Trauma and Orthopaedic Surgery Service, Hospital de Sant Joan Moises Broggi, Jacint Verdaguer 90 , Sant Joan Despi 08970, Barcelona, Spain

Tel +34 935531200

Email eloisa.velasco@sanitatintegral.org
Introduction: Osteoarthritis of the trapeziometacarpal (TMC) joint of the thumb - also known as rhizarthrosis - is painful and has a significant impact on quality of life. Intra-articular injection of hyaluronic acid may potentially meet the need for effective, minimally invasive intervention in patients not responding adequately to initial treatment. We aimed to confirm the safety and effectiveness of viscosupplementation with Durolane (NASHA nonanimal hyaluronic acid) in rhizarthrosis. Patients and methods: This was a prospective, single-arm, multicenter, open-label study with a 6-month follow-up period. Eligible patients had Eaton-Littler grade II-III rhizarthrosis in one TMC joint with pain and visual analog scale (VAS) pain score $\geq 4$ (scale: $0-10$ ). A single injection of NASHA was administered to the affected TMC joint. The primary effectiveness variable was change from baseline in VAS pain score.

Results: Thirty-five patients (mean age 60.8 years; $85.7 \%$ female) received NASHA and completed the study. The least-squares mean change from baseline in VAS pain score over 6 months was -2.00 , a reduction of $27.8 \%(p<0.001)$. The reduction in pain exceeded $25 \%$ as early as month 1 (26.5\%), and gradual improvement was observed throughout the 6-month follow-up period. Secondary effectiveness parameters included QuickDASH (shortened version of Disabilities of the Arm, Shoulder, and Hand [DASH]), Kapandji thumb opposition test, radial abduction, metacarpophalangeal (MCP) joint flexion, and pinch (clamp) strength. Most of these measurements showed statistically significant improvements from baseline over 6 months. Five adverse events (injection site reactions) were reported in four patients (11.4\%), and there were no serious or allergic reactions.

Conclusion: This study suggests that viscosupplementation using NASHA is effective and well tolerated in treating the symptoms of rhizarthrosis.

Keywords: Durolane, hyaluronic acid, osteoarthritis, pain, rhizarthrosis, viscosupplementation

\section{Introduction}

Radiographic osteoarthritis (OA) of the hand is detectable in over $50 \%$ of individuals aged over 55 years. ${ }^{1}$ The prevalence of hand OA increases with age, and it is more common in women than in men. The condition often becomes painful, and hand pain has been reported to affect approximately one in six individuals aged over 55 years. ${ }^{2}$ Musculoskeletal hand problems also cause significant impairment of daily activities and reduced satisfaction with hand appearance. ${ }^{3}$ The effects on everyday life are likely to be greatest in women and the very elderly. There is therefore a considerable need for effective treatment options.

Intra-articular injection of hyaluronic acid (HA) has the potential to meet the need for effective, minimally invasive intervention in patients not responding adequately to 
first-line treatment options. NASHA (nonanimal hyaluronic acid) is approved in Europe for the treatment of small-joint OA, including rhizarthrosis. It is manufactured using nonanimal hyaluronic acid. During the manufacturing process, the natural entanglements that exist in normal HA are supplemented by a limited extent of synthetic cross-linking $(\sim 1 \%)$ to create a three-dimensional gel network. The end result is a viscous gel with increased density of HA, but unaltered characteristics of the polysaccharide chain. NASHA resides in the joint for longer than other HA preparations: in a rabbit knee model, the half-life for unmodified HA is reported to be less than 24 hours, ${ }^{4}$ whereas the half-life of NASHA is 32 days. ${ }^{5}$ In comparison, the half-lives of the components of Synvisc (Hylan-A, the fluid; and Hylan-B, the gel) are 1.5 days and 8.8 days, respectively. ${ }^{6}$

We performed a single-arm study to confirm the effectiveness of viscosupplementation with NASHA in the treatment of rhizarthrosis. The study hypothesis was that visual analog scale (VAS) pain over months 1,3 , and 6 would be reduced by $25 \%$ from baseline.

\section{Patients and methods}

This was a prospective, single-arm, open-label study with 6 months follow-up, performed across three sites in Barcelona, Spain. It was performed in accordance with Good Clinical Practice and the Declaration of Helsinki. The study protocol was approved by the ethics committee at each center: Comite Etico de Investigación Clínica del Consorci Sanitari Integral (Hospital Moisés Broggi), Comité Etico de Investigación Clínica del Hospital Parc Taulí, and Comité Etico de Investigación Clínica del Hospital Universitario de la Vall d'Hebron.

Patients aged 18-75 years with rhizarthrosis in either hand were eligible for inclusion in the study. Rhizarthrosis had to be diagnosed radiologically in the study hand as grade II or III according to established criteria. ${ }^{7}$ In addition, all study participants were required to have trapeziometacarpal (TMC) joint pain in the study hand of more than 6 months' duration with a VAS pain score of at least 4 (scale: 0 , absence of pain, to 10, maximum pain imaginable) and a VAS pain score below 4 in the contralateral hand. The main exclusion criteria were anticoagulant medication; rheumatic disease such as rheumatoid arthritis or gout involving the wrist, hand, or fingers; previous surgery of the hand; systemic infectious disease; and previous HA injections in the hand.

Each patient received a single injection of Durolane (NASHA nonanimal hyaluronic acid; Bioventus LLC, Durham, NC, USA; $20 \mathrm{mg} / \mathrm{mL}$ sodium hyaluronate in a prefilled $1 \mathrm{~mL}$ syringe) into the affected TMC joint. Fluoroscopy (double projection without contrast) was used to ensure injection into the correct joint. The aim was to inject the entire syringe volume of $1 \mathrm{~mL}$, but injections were stopped early (eg, after 0.7 or $0.8 \mathrm{~mL}$ ) in case of difficulties such as increased resistance. Follow-up clinic visits were scheduled 1,3 , and 6 months posttreatment for assessment of effectiveness and safety. No analgesics or anti-inflammatories were permitted 24 hours before scheduled clinical visits.

Effectiveness was assessed principally using the VAS pain score. Patients also completed the QuickDASH questionnaire, a shortened version of Disabilities of the Arm, Shoulder, and Hand (DASH) designed to measure physical function and symptoms in the context of musculoskeletal disorders of the upper limb. QuickDASH provides scores ranging between 11 and 55; these were remapped to a $0-100$ scale. The Kapandji thumb opposition test (score range: 0-10), radial abduction (flexion angle, measured in degrees), and metacarpophalangeal (MCP) joint flexion (measured in degrees) provided specific indications of the range of thumb movement. Pinch (clamp) strength was measured using a pinch gauge to find the strength of lateral pinch between the thumb and second finger, and fist strength was measured using a hand dynamometer. As additional effectiveness parameters, the presence or absence of crepitus, morning stiffness, and mobility difficulties were recorded. All concomitant medications taken during the study were recorded. To assess safety, adverse events (AEs) were reported throughout the study. A physical examination was performed at each clinic visit; notable findings were recorded as AEs.

\section{Statistical methods}

The primary effectiveness variable was change from baseline in VAS pain score. This was analyzed using mixed-effect repeated measures regression, with VAS pain score as the dependent variable. Fixed-effect covariates were centers 1 , 2 , and 3 and months $0,1,3$, and 6; subject was the random effect. The criteria for study success were least-squares mean VAS pain scores for months 1, 3, and 6 at least $25 \%$ below the least-squares mean VAS pain score at month 0 (baseline). This threshold was selected for consistency with the minimum clinically important improvement in VAS score of $25 \% .{ }^{8}$ The significance level for a type I error was 0.05 . For detection of a $25 \%$ change in VAS pain score with a power of 0.80 , a sample of 35 patients was considered to be suitable.

Changes in secondary effectiveness variables were analyzed using the same mixed-effects repeated measures regression model used for the primary VAS pain assessment. Effectiveness was assessed in the full analysis set, comprising 
all patients receiving study treatment with at least one posttreatment effectiveness assessment. Safety was assessed in the safety set: all patients receiving study treatment.

\section{Results}

\section{Patients}

Thirty-six patients provided signed, informed consent, of whom 35 received study treatment (one patient withdrew consent before receiving study treatment). All 35 patients who received study treatment completed the study. Therefore, both the full analysis set and the safety set comprised 35 patients. One patient missed their month 3 visit, and in another patient data from visits at months 5 and 7 visit were averaged and used for month 6 .

The mean age of the full analysis set was 60.8 years, and $85.7 \%$ of the study participants were female. Patients' baseline characteristics are summarized in Table 1; the mean

Table I Patient demographics and baseline characteristics (full analysis set, $\mathrm{N}=35$ )

\begin{tabular}{ll}
\hline Characteristics & Mean (SD) \\
& or $\mathbf{n ~ ( \% )}$ \\
\hline Age (years) & $60.8(8.3)$ \\
Female gender & $30(85.7)$ \\
Body weight $(\mathrm{kg})$ & $69.2(\mathrm{I} 2.3)$ \\
Body mass index $\left(\mathrm{kg} / \mathrm{m}^{2}\right)$ & $26.9(4.1)$ \\
VAS pain score: treated hand & $7.2(\mathrm{I} .8)$ \\
VAS pain score: contralateral hand & $2.2(\mathrm{I} .4)$ \\
Duration of rhizarthrosis (years) & $7.1(5.6)$ \\
Rhizarthrosis classification (Eaton-Littler grade) & \\
II & $14(40.0 \%)$ \\
III & $21(60.0 \%)$ \\
\hline Abbreviations: SD, standard deviation; VAS, visual analog scale. &
\end{tabular}

duration of rhizarthrosis was 7.1 years, and $60 \%$ of patients had grade III rhizarthrosis. The mean VAS pain score in the treated hand was 7.2.

\section{Primary effectiveness analysis}

The least-squares mean change from baseline in VAS pain score over 6 months was -2.00 , a reduction of $27.8 \%$ $(p<0.001)$ from the mean baseline value of 7.2 (Table 2$)$. The mean percentage decrease versus baseline was greater than $25 \%$ at all three assessment time-points, with continuous improvement throughout the study period (month 1 , $26.5 \%$; month $3,28.7 \%$; month $6,32.6 \%$ ). Therefore, the primary study success criteria were met. The mean VAS pain score at each study time-point and percentages of patients with at least $25 \%$ decrease from baseline are also shown in Table 2.

\section{Secondary effectiveness analyses}

Reflecting the primary analysis, the QuickDASH questionnaire results showed continuous improvement during the study (Table 3). Mean percentage improvements in the QuickDASH score were $14.0 \%, 15.7 \%$, and $22.9 \%$ at months 1,3 , and 6 , respectively. The difference versus baseline over 6 months was statistically significant $(p<0.001)$. A positive response to NASHA was evident from the Kapandji thumb opposition test results (Table 3). Although this parameter did not show continuous improvement over time, the largest improvement (mean 16.1\%) was evident at the 6-month time-point, and the change from baseline over 6 months was statistically significant $(p=0.0114)$. Improvements versus

Table 2 VAS pain scores: primary effectiveness analysis

\begin{tabular}{|c|c|c|c|c|c|}
\hline Visit & Statistic & Actual & Change & $\%$ Change & $\begin{array}{l}\geq 25 \% \text { reduction } \\
\text { versus baseline }\end{array}$ \\
\hline \multirow[t]{3}{*}{ Baseline } & $\mathrm{N}$ & 35 & & & \\
\hline & Mean (SD) & $7.2(1.8)$ & & & \\
\hline & Min-Max & $4.5-10.0$ & & & \\
\hline \multirow[t]{3}{*}{ Month I } & $\mathrm{N}$ & 35 & 35 & 35 & 35 \\
\hline & Mean (SD) & $5.4(2.5)$ & $-1.8(1.8)$ & $-26.5(26.9)$ & $17(48.6 \%)^{\mathrm{a}}$ \\
\hline & Min-Max & $1.0-10.0$ & -6.0 to 1.0 & -80.0 to 20.0 & \\
\hline \multirow[t]{3}{*}{ Month 3} & $\mathrm{~N}$ & 34 & 34 & 34 & 34 \\
\hline & Mean (SD) & $5.3(2.7)$ & $-1.9(1.9)$ & $-28.7(31.0)$ & $19(55.9 \%)^{\mathrm{a}}$ \\
\hline & Min-Max & $1.0-10.0$ & -6.0 to 2.0 & -83.3 to 33.3 & \\
\hline \multirow[t]{3}{*}{ Month 6} & $\mathrm{~N}$ & 35 & 35 & 35 & 35 \\
\hline & Mean (SD) & $5.0(2.7)$ & $-2.2(2.0)$ & $-32.6(31.0)$ & $19(54.3 \%)^{\mathrm{a}}$ \\
\hline & Min-Max & $1.0-10.0$ & -6.0 to 0.8 & -81.8 to 16.0 & \\
\hline \multirow[t]{3}{*}{ Over 6 months } & LS mean (SE) & $-2.00(0.28)$ & & & \\
\hline & $95 \% \mathrm{Cl}$ & -2.57 to -1.4 & & & \\
\hline & t statistic ( $P$-value $)$ & $-7.14(P<0.0001)$ & & & \\
\hline
\end{tabular}

Notes: ${ }^{a}$ Data shown are number of patients (\%).

Abbreviations: $\mathrm{Cl}$, confidence interval; LS, least square; SD, standard deviation; SE, standard error; VAS, visual analog scale. 
Table 3 Secondary effectiveness results: mean (SD) or $\mathrm{n}(\%)$

\begin{tabular}{lllll}
\hline Parameter & Baseline $(\mathbf{N}=35)$ & I month $(\mathbf{N}=35)$ & 3 months $(\mathbf{N}=34)$ & 6 months $(\mathbf{N}=35)$ \\
\hline QuickDASH total score & $58.0(16.9)$ & $49.4(18.4)$ & $49.1(22.9)$ & $44.5(21.3)$ \\
Kapandji score & $7.2(2.1)$ & $7.7(1.9)$ & $7.6(1.9)$ & $8.1(1.8)$ \\
Radial abduction score & $28.9(13.8)$ & $32.5(12.1)$ & $34.5(11.9)$ & $34.1(14.9)$ \\
MCP flexion score & $42.8(21.7)$ & $46.9(21.2)$ & $49.2(19.0)$ & $48.0(17.8)$ \\
Strength of fist $(\mathrm{kg})$ & $10.8(6.9)$ & $11.6(6.1)$ & $11.6(5.6)$ & $13.0(5.9)$ \\
Strength of clamp $(\mathrm{kg})$ & $3.1(3.4)$ & $4.5(8.1)$ & $3.0(3.1)$ & $5.1(5.0)$ \\
Crepitus present & $17(48.6 \%)$ & $1 \mathrm{I}(31.4 \%)$ & $7(20.6 \%)$ & $10(28.6 \%)$ \\
Stiffness present & $16(45.7 \%)$ & $10(28.6 \%)$ & $8(23.5 \%)$ & $9(25.7 \%)$ \\
Mobility difficulties present & $20(57.1 \%)$ & $16(45.7 \%)$ & $14(41.2 \%)$ & $14(40.0 \%)$ \\
\hline
\end{tabular}

Abbreviations: DASH, disabilities of the arm, shoulder and hand; MCP, metacarpophalangeal; SD, standard deviation.

baseline were observed in radial abduction, MCP flexion, strength of fist, and strength of clamp scores (Table 3). For all of these parameters except strength of fist, the change from baseline over 6 months was statistically significant $(p<0.05)$. These parameters did not show continuous improvement over time, although mean percentage improvements in strength of fist and strength of clamp were greatest at 6 months (46.2\% and $136.0 \%$, respectively). Maximum percentage improvements in radial abduction score $(46.8 \%)$ and MCP flexion score (40.3\%) were observed at 3 months.

Percentages of patients with crepitus, stiffness, and mobility difficulties reduced considerably in response to NASHA treatment (Table 3 ). The percentage of patients with mobility difficulties decreased continuously with time, from $57.1 \%$ at baseline to $40 \%$ at the 6-month time-point.

Regarding concomitant medications, the requirement for a 24-hour washout period before clinic visits was met by all patients at baseline, 3 months, and 6 months. All except two patients adhered to this requirement before the 1-month visit. Analgesic medication was taken by 11 patients (31.4\%) after NASHA treatment, the indications being pain $(n=5)$, low back pain $(n=4)$, shoulder pain $(n=1)$, and musculoskeletal pain $(n=1)$. The most commonly used analgesic medications were paracetamol $(n=7)$, tramadol $(n=4)$, and ibuprofen $(n=3)$. Overall, concomitant drug use was not considered to have confounded the effectiveness results.

\section{Safety}

A total of five AEs were reported in four patients (11.4\% of the safety population) (Table 4 ). All five AEs occurred on day 1. None were classified as serious or interpreted as allergic reactions. Two of the AEs occurred in one patient: pain and swelling. Both of these AEs were of moderate intensity and both were related to study treatment. Three out of the four patients with AEs were treated with analgesic medication
Table 4 Listing of AEs

\begin{tabular}{lll}
\hline Description & $\begin{array}{l}\text { Relationship with } \\
\text { study treatment }\end{array}$ & Intensity \\
\hline Increased pain & Probable & Severe \\
Pain & Possible & Moderate \\
Swelling & Possible & Moderate \\
Inflammation & Definite & Moderate \\
Pain & Possible & Moderate \\
\hline
\end{tabular}

Notes: Each row in the table represents a single AE in one patient. Pain and swelling were coexistent in one patient; the remaining events all occurred in different patients. Thus, there were five AEs in four patients.

Abbreviation: $A E$, adverse event.

or nonsteroidal anti-inflammatory drugs, and all of the AEs except one resolved within a week. The exception was pain in one patient that lasted for 95 days.

\section{Discussion}

This study of intra-articular NASHA for rhizarthrosis suggested that the treatment is well tolerated and effective in reducing symptoms. VAS pain score improved significantly, with a clinically meaningful $25 \%$ reduction in pain exceeded throughout the 6-month follow-up period. A positive response to viscosupplementation was also evident in joint function (QuickDASH and Kapandji scores) and biomechanical function (radial abduction, MCP flexion, strength of clamp). The only one of these parameters for which improvement over 6 months did not reach statistical significance was fist strength. This test does not directly involve the thumb joint and is therefore not a direct reflection of treatment effectiveness.

AEs were reported in only a minority of patients, and all were resolved during the study. The nature of the observed AEs was as expected: injection site reactions that are common with any intra-articular injection therapy. No safety concerns emerged during the study.

OA cannot currently be cured and, consequently, pain therapy is an important medical need. Intra-articular injection 
of HA is intended to relieve pain. The antinociceptive properties of NASHA were investigated by Boettger et $\mathrm{al}^{9}$ using an animal model in which pain was created by injecting joints with bradykinin and prostaglandin E2, both of which are found in OA. Animals treated with saline responded to around $160 \mathrm{~g}$ of pressure on the joint, whereas those treated with NASHA accepted around $240 \mathrm{~g}$. These data show that NASHA is effective for inhibiting the sensation of pain. The exact mechanism of action is currently unknown, but several possibilities have been postulated (eg, mechanical lubrication/protection of the joint; coverage of sensory endings; molecular binding of pain mediators; fulfillment of trophicmetabolic functions, etc).

Previous studies of viscosupplementation for rhizarthrosis have reported promising results. ${ }^{10-13}$ The first clinical trial to report such data was a 26-week, randomized, prospective comparison of sodium hyaluronate with triamcinolone acetonide in 56 patients with OA of the carpometacarpal joint of the thumb. ${ }^{12}$ Three injections were administered to each patient. Although triamcinolone acetate provided significantly better pain relief than sodium hyaluronate at 2-3 weeks (median VAS pain score 20.0 versus $34.0 \mathrm{~mm}$ ), pain relief reached a maximum with sodium hyaluronate at week 26 , at which time a trend toward superiority versus triamcinolone acetate was evident (30.0 versus $45.5 \mathrm{~mm}$ ). Accordingly, lateral pinch power was significantly higher with sodium hyaluronate at 26 weeks. In a subsequent double-blind controlled trial, 60 patients with basal joint OA were randomized to receive two injections of Hylan G-F 20, one saline injection followed by one corticosteroid injection, or two saline injections. ${ }^{13}$ Statistically significant between-group differences in pain were not observed, but significant improvements in pain versus baseline at weeks 12 and 26 were observed only in the Hylan G-F 20 group. Thirty-three women with bilateral thumb base OA participated in another study of Hylan G-F 20. ${ }^{11}$ Each patient received treatment with a single injection of Hylan G-F 20 in one hand, and saline in the other hand. Statistically significant improvements at 24 weeks versus baseline were evident with Hylan G-F 20 in relation to VAS pain score, pinch strength, and functionality (Dreiser functional index). In contrast, saline injection only produced a temporary improvement ( 6 weeks) in VAS pain score. In a fourth study, 40 women were randomized 1:1 to receive compared sodium hyaluronate or triamcinolone acetate as a single injection into the TMC joint. ${ }^{10}$ VAS pain score decreased significantly over 12 months with triamcinolone acetate and over 6 months with sodium hyaluronate. Hand function (Duruöz Hand Index) showed improvement in both study groups, although this reached statistical significance only in the triamcinolone acetate group.

Our results are consistent with studies of intra-articular NASHA injection for knee and hip OA, ${ }^{14-18}$ showing a long duration of action and favorable tolerability. For example, a 26-week study comparing single-injection NASHA with methylprednisolone acetate in knee OA showed similar Western Ontario and McMaster Universities (WOMAC) OA pain index responder rates in the two study groups at 12 weeks, with the WOMAC pain effect size increasingly favoring NASHA between 12 and 26 weeks. ${ }^{18}$ In hip OA, a WOMAC pain response rate of $54 \%$ has been reported 3 months after a single injection of NASHA, with as many as two-thirds of 3-month responders continuing to show response at 6-11 months. ${ }^{16}$ All of these studies reported favorable tolerability of NASHA, with mostly transient, local adverse effects relating to the injection procedure and a lack of allergic reactions or safety concerns.

One limitation of the present study is the lack of a control group - randomized controlled trials are needed to provide more robust evidence of the effectiveness of NASHA for rhizarthrosis. In the absence of such data, the clinical relevance of the observed reduction in VAS pain score may, potentially, be questioned. Another limitation was the lack of requirement for physical examination when diagnosing rhizarthrosis, potentially reducing the reliability of diagnosis. Strengths of the study include fulfillment of the number of patients required for statistical power and recruitment of patients with only minor symptoms in the contralateral joint, thereby ensuring sensitivity to treatment response. In addition, the inclusion of a variety of effectiveness endpoints demonstrated the comprehensive nature of the response to treatment.

\section{Conclusion}

This study suggests that viscosupplementation using NASHA is effective in treating the symptoms of rhizarthrosis, with improvements observed across a range of different effectiveness parameters. In accordance with previous NASHA studies, the treatment was also shown to be well tolerated.

\section{Acknowledgments}

The authors would like to thank the remainder of the Durolane study group for their valuable contributions to this study: R Calonge, M Lafuente (Department of Orthopaedic Surgery, Hospital de Sant Joan Despí Moisés Broggi, 
Barcelona, Spain); J Medel, A Mesas, E Márquez, A Candela, P Martinez, M Paños (Department of Anesthesiology, Vall d'Hebron University Hospital, Barcelona, Spain); R Sánchez, A Berenguer, F Fillat, and C Villamil (Department of Orthopedics and Traumatology, Parc Taulí University Hospital, Sabadell, Barcelona, Spain). This study was sponsored by Zambon SAU, Barcelona, Spain, and Bioventus LLC, Durham, NC, USA. Both sponsors worked with the authors in developing the study design. Statistical analysis and preparation of the study report were performed by Bioventus LLC. The authors thank Ken Sutor who provided medical writing services on behalf of Bioventus LLC.

\section{Author contributions}

EV, MVR, and JP contributed to the study design, recruited and treated patients, approved the statistical analysis and study report, edited the manuscript, and approved the final version.

\section{Disclosure}

The authors report no conflicts of interest in this work.

\section{References}

1. Dahaghin S, Bierma-Zeinstra SM, Ginai AZ, Pols HA, Hazes JM, Koes BW. Prevalence and pattern of radiographic hand osteoarthritis and association with pain and disability (the Rotterdam study). Ann Rheum Dis. 2005;64(5):682-687.

2. Dahaghin S, Bierma-Zeinstra SM, Reijman M, Pols HA, Hazes JM, Koes BW. Prevalence and determinants of one month hand pain and hand related disability in the elderly (Rotterdam study). Ann Rheum Dis. 2005;64(1):99-104.

3. Dziedzic K, Thomas E, Hill S, Wilkie R, Peat G, Croft PR. The impact of musculoskeletal hand problems in older adults: findings from the North Staffordshire Osteoarthritis Project (NorStOP). Rheumatology (Oxford). 2007;46(6):963-967.

4. Brown TJ, Laurent UB, Fraser JR. Turnover of hyaluronan in synovial joints: elimination of labelled hyaluronan from the knee joint of the rabbit. Exp Physiol. 1991;76(1):125-134.
5. Edsman $\mathrm{K}, \mathrm{Hjelm} \mathrm{R}$, Larkner $\mathrm{H}$, et al. Intra-articular duration of Durolan$\mathrm{e}^{\mathrm{TM}}$ after single injection into the rabbit knee. Cartilage. 2011;2:384-388.

6. Larsen NE, Dursema HD, Pollak CT, Skrabut EM. Clearance kinetics of a hylan-based viscosupplement after intra-articular and intravenous administration in animal models. J Biomed Mater Res B Appl Biomater. 2012;100(2):457-462.

7. Eaton RG, Lane LB, Littler JW, Keyser JJ. Ligament reconstruction for the painful thumb carpometacarpal joint: a long-term assessment. J Hand Surg Am. 1984;9(5):692-699.

8. Dworkin RH, Turk DC, Wyrwich KW, et al. Interpreting the clinical importance of treatment outcomes in chronic pain clinical trials: IMMPACT recommendations. J Pain. 2008;9(2):105-121.

9. Boettger MK, Kummel D, Harrison A, Schaible HG. Evaluation of longterm antinociceptive properties of stabilized hyaluronic acid preparation (NASHA) in an animal model of repetitive joint pain. Arthritis Res Ther. 2011;13(4):R110.

10. Bahadir C, Onal B, Dayan VY, Gurer N. Comparison of therapeutic effects of sodium hyaluronate and corticosteroid injections on trapeziometacarpal joint osteoarthritis. Clin Rheumatol. 2009;28(5):529-533.

11. Figen Ayhan F, Ustun N. The evaluation of efficacy and tolerability of Hylan G-F 20 in bilateral thumb base osteoarthritis: 6 months followup. Clin Rheumatol. 2009;28(5):535-541.

12. Fuchs S, Monikes R, Wohlmeiner A, Heyse T. Intra-articular hyaluronic acid compared with corticoid injections for the treatment of rhizarthrosis. Osteoarthritis Cartilage. 2006;14(1):82-88.

13. Heyworth BE, Lee JH, Kim PD, Lipton CB, Strauch RJ, Rosenwasser MP. Hylan versus corticosteroid versus placebo for treatment of basal joint arthritis: a prospective, randomized, double-blinded clinical trial. J Hand Surg Am. 2008;33(1):40-48.

14. Altman RD, Akermark C, Beaulieu AD, Schnitzer T; Durolane International Study G. Efficacy and safety of a single intra-articular injection of non-animal stabilized hyaluronic acid (NASHA) in patients with osteoarthritis of the knee. Osteoarthritis Cartilage. 2004;12(8):642-649.

15. Arden NK, Akermark C, Andersson M, Todman MG, Altman RD. A randomized saline-controlled trial of NASHA hyaluronic acid for knee osteoarthritis. Curr Med Res Opin. 2014;30(2):279-286.

16. Berg P, Olsson U. Intra-articular injection of non-animal stabilised hyaluronic acid (NASHA) for osteoarthritis of the hip: a pilot study. Clin Exp Rheumatol. 2004;22(3):300-306.

17. Conrozier T, Couris CM, Mathieu P, et al. Safety, efficacy and predictive factors of efficacy of a single intra-articular injection of non-animalstabilized-hyaluronic-acid in the hip joint: results of a standardized follow-up of patients treated for hip osteoarthritis in daily practice. Arch Orthop Trauma Surg. 2009;129(6):843-848.

18. Leighton R, Akermark C, Therrien R, et al. NASHA hyaluronic acid vs. methylprednisolone for knee osteoarthritis: a prospective, multicentre, randomized, non-inferiority trial. Osteoarthritis Cartilage. 2014; 22(1):17-25.
Open Access Rheumatology: Research and Reviews

\section{Publish your work in this journal}

Open Access Rheumatology: Research and Reviews is an international, peerreviewed, open access journal publishing original research, reports, editorials, reviews and commentaries on all aspects of clinical and experimental rheumatology in the clinic and laboratory including the following topics: Pathology, pathophysiology of rheumatological diseases; Investigation, treatment and

\section{Dovepress}

management of rheumatological diseases; Clinical trials and novel pharmacologi$\mathrm{cal}$ approaches for the treatment of rheumatological disorders. The manuscript management system is completely online and includes a very quick and fair peer-review system, which is all easy to use. Visit http://www.dovepress.com/ testimonials.php to read real quotes from published authors. 